\title{
Inhibition of autophagy by 3-MA enhances IL-24-induced apoptosis in human oral squamous cell carcinoma cells
}

Jichen Li $i^{1}$, Dezhao Yang ${ }^{2}$, Wei Wang ${ }^{2}$, Songlin Piao ${ }^{2}$, Jianyu Zhou ${ }^{2}$, Wuliji Saiyin² ${ }^{2}$ Changyu Zheng ${ }^{3}$, Hongchen $\mathrm{Sun}^{4^{*}}$ and $\mathrm{Yu} \mathrm{Li}{ }^{1^{*}}$

\begin{abstract}
Background: Interleukin-24(IL-24), also referred to as melanoma differentiation-associated gene-7(mda-7), is a unique member of the IL-10 gene family, which displays nearly ubiquitous cancer-specific toxicity. The most notable feature of IL-24 is selectively induced growth suppression and apoptosis in various cancer cells, with no harmful effects toward normal cells. Autophagy is a self-protective mechanism in many kinds of tumor cells that respond to anticancer treatment. It is reported that autophagy inhibition could enhance the effects of many kinds of anticancer treatments, including gene therapy. However, whether IL-24 is effective to treat oral squamous cell carcinomas (OSCC) and if autophagy inhibition could improve the anticancer effect of IL-24 towards OSCC is has not been detected.

Methods: MTT assays were carried out to determine the cell proliferation; Transfection was used to gene transfer; Western Blot was performed to detect the protein level of LC3II, P62, Beclin 1, Cleaved caspase-3, $\beta$-Tubulin and $\beta$-actin; Apoptosis rates and cell cycle alteration were analyzed using flow cytometry; Autophagy induction was confirmed by MDC staining, GFP-LC3 staining and transmission electron microscopy. Amount of IL-24 in the culture medium was quantified by ELISA. Apoptosis in vivo was analyzed by TUNEL assay. HE staining was used to observe the morphology of the samples.
\end{abstract}

Results: In the present study, we proved that IL-24 have a novel anticancer effect towards KB cells and that autophagy inhibition could improve the anticancer effect of IL-24. IL-24 treated cells showed autophagy characteristics and autophagy inhibition by 3-methyladenine (3-MA) significantly enhanced IL-24-induced apoptosis. Similar results were obtained in the KB cells xenograft tumor model.

Conclusions: These results suggest that the combination of autophagy inhibitors and IL-24 based on the AdLTR2EF1amediated gene transfer could be a promising way to cure OSCC.

Keywords: OSCC, Interleukin-24, Autophagy inhibition, Apoptosis, Gene therapy

\section{Background}

Oral cancer is a widespread malignant disease, with over 640,000 new cases being found annually worldwide. There are several types of oral cancers, but approximately than $90 \%$ are squamous cell carcinomas. The prognosis of OSCC is poor due to their strong invasive nature [1]. While the effect of conventional treatments, such as

\footnotetext{
* Correspondence: hcsun@mail.ju.edu.cn; liyugene@hit.edu.cn

${ }^{4}$ Department of Oral Pathology, School of Stomatology, Jilin University, 1500

Qinghua Road, Changchun 130021, People's Republic of China

'School of Life Science and Technology, Harbin Institute of Technology, 2

Yikuang Street, Harbin 150001, People's Republic of China

Full list of author information is available at the end of the article
}

surgical removal, chemotherapy and radiotherapy is limited for oral cancer. Recent years, gene therapy known as a new way of treating cancer have attracted increasing interest. Gene therapy describes the delivery of a functional therapeutic gene to target cells, which may be used to knockdown expression of a particular macromolecule, improve the level of desired protein, directly induce cell death, or replace a defective or mutant gene to allow a normal protein product to a certain level. mda-7/ IL-24 is an unusual member of the IL-10 cytokine family [2], with ubiquitous tumor cell proapoptotic activity [3]. Recent reports have demonstrated the successful entry of 
mda-7/IL-24 into the clinic with safety and clinical efficacy when administered by adenovirus [4]. Despite extensive studies, questions still remain about how to further enhance the anti-tumor effect of IL-24.

Recently, the elegant concept of autophagy inhibition provides a new strategy for efficient cancer therapy. In general, when the cells respond to the limited nutrition and growth factors, autophagy is activated and contributes to maintaining homeostasis through degradation of impaired or unnecessary macromolecules and organelles, thereby providing energy to cancer cells [5]. Autophagy usually serves as a protective mechanism for tumor cells exposed to anticancer treatments [6]. In preclinical trials, repression of autophagy has demonstrated an increased efficacy of chemotherapeutics, both in vitro and in vivo [7-10]. Recent studies have shown that IL-24 induces endoplasmic reticulum stress response via induction of autophagy in glioblastoma cells through PERK activation [11]. However, whether autophagy inhibition can enhance the acticancer effects of IL-24 in treating oral cancer is have not been investigated.

In this study, we utilized a novel hybrid gene delivery vector named $\mathrm{AdLTR}_{2} \mathrm{EF} 1 \alpha$-based vector, which we have constructed in our previous work [12], as a gene carrier of IL-24 to treat $\mathrm{KB}$ (human Oral epidermoid cancer cells) and $\mathrm{HaCaT}$ (immortal human keratinocyte cells) cell lines. High level of apoptosis as well as autophagy were observed in $\mathrm{AdLTR}_{2} \mathrm{EF} 1 \alpha$-IL-24 treated cells. To our surprise, while the autophagy induced by AdLTR $_{2} E F 1 \alpha-I L-24$ was blocked by autophagy inhibitor 3-MA, a significant increase of anticancer effect was detected. Similar results were obtained in KB xenografts in nude mice. This work highlights the potential of combination of IL-24 gene and autophagy inhibitor for enhanced efficacy against aggressive oral cancer.

\section{Methods}

\section{Cell lines and cell cultures}

In this study we used $\mathrm{KB}$ cells and $\mathrm{HaCaT}$ cells (control). KB cells were cultured in RPMI 1640 medium (Gibco, USA) and $\mathrm{HaCaT}$ cells were cultured in DMEM medium (Gibco, USA). All medium was supplemented with $10 \%$ fetal bovine serum (Gibco, USA), and $1 \%$ penicillin and streptomycin at $37{ }^{\circ} \mathrm{C}$ in $5 \% \mathrm{CO}_{2}, 95 \%$ humidified incubator.

\section{AdLTR $_{2}$ EF1a-mediated gene transfer}

In order to assess the appropriate transfection concentration, $\mathrm{KB}$ and $\mathrm{HaCaT}$ cells were infected with $\mathrm{AdLTR}_{2} \mathrm{EF} 1 \alpha-$ vec, at different concentrations. Cell viability was assessed by MTT $72 \mathrm{~h}$ after infection. After determining the optimal transfection concentration, $\mathrm{KB}$ cells and $\mathrm{HaCaT}$ cells were infected with AdLTR $_{2}$ EF1 $\alpha$-EGFP at 1000 pfu/cell. Enhanced level of green fluorescent protein (EGFP) was examined by fluorescence microscopy at 12, 24 and $48 \mathrm{~h}$ after infection. Expression of transgenic IL-24 in $\mathrm{KB}$ and $\mathrm{HaCaT}$ cells was determined by real time reversetranscription polymerase chain reaction (real time RTPCR) $48 \mathrm{~h}$ after infection. Total RNA was extracted using RNeasy mini purification kit (Qiagen, USA). RNA was quantitated using a NanoDrop2000 spectrophotometer (Thermo, USA). Complementary DNA was synthesized with reverse transcriptase (TaKaRa, Japan), The qPCRs were performed using SYBR-Green premix Ex Taq (Takara) $(n=3)$ and MxPro Mx3005P real-time PCR detection system (Agilent, USA). IL-24 gene was amplified using the specific primers forward 5 - AAGCCTGTGGACTTTAGC CAGACC -3 ' and reverse, 5'- GCACTCGTGATGTTAT CCTGAGC $-3{ }^{\prime}$. $\beta$-actin primers forward $5^{\prime}-$ TGGCACC CAGCACAATGAA -3', and reverse 5'- CTAAGTCA TAGTCCGCCTAGAAGCA -3' for human $\beta$-actin amplicon as internal control. Primers were synthesized commercially (TaKaRa Biotechnology [Dalian] Co). Furthermore, IL-24 in the culture medium of all samples was quantified by sandwich enzyme-linked immunosorbent assay (ELISA) kits (Lengton, China).

\section{Measurement of autophagy}

KB cells were infected with AdLTR $_{2} E F 1 \alpha-I L-24$ or AdLTR $_{2} E F 1 \alpha$-vec. Before each time point, cells were cultured with $50 \mu \mathrm{M}$ Monodansylcadaverine (MDC) for $1 \mathrm{~h}$, and then trypsinized. Trypsinized cells were collected, resuspended, dropped onto a glass slide and examined under fluorescence microscopy in UV channels. Additional cells were transfected with green fluorescent protein (GFP)-labeled LC3 fusion protein following infection with $\mathrm{AdLTR}_{2} \mathrm{EF} 1 \alpha-\mathrm{IL}-24$. Cells was examined at different time points after infection using fluorescence microscopy. At each time point, cells in each group were collected and washed with cold PBS, then $0.25 \%$ neutral glutaraldehyde fixed overnight at $4{ }^{\circ} \mathrm{C}$ for TEM sectioning. Subsequently, the cellular microstructure was observed by the transmission electron microscope (TEM).

\section{Western blotting}

KB cells infected with AdLTR ${ }_{2} \mathrm{EF} 1 \alpha$-IL-24 or $\mathrm{AdLTR}_{2} \mathrm{EF} 1 \alpha-$ vec were cultured at $37{ }^{\circ} \mathrm{C}$ for $48 \mathrm{~h}$. Cells were harvested and resuspended in lysis buffer containing $50 \mathrm{mM}$ Tris- $\mathrm{HCl}$ (pH7.4), $150 \mathrm{mM} \mathrm{NaCl}, 1 \%$ Triton X-100, 1 \% sodium deoxycholate, $0.1 \%$ SDS and $1 \%$ protease inhibitor cocktail. Protein from each sample was separated by SDS-PAGE and PVDF membranes. The membranes were blocked with $5 \%$ skim milk, primary antibodies diluted with TBS-T by 1:1000 and incubated with primary antibody at $4{ }^{\circ} \mathrm{C}$ for $12 \mathrm{~h}$, followed by incubation with secondary antibody diluted with TBS-T for $1 \mathrm{~h}$. LC3-II antibody purchased from Abcam company (USA). The protein levels of LC3-II were detected by Odyssey Two-Color Infrared Imaging System 
(the secondary antibody was labeled by FITC fluorescence). $\beta$-tubulin was used as a loading control. IL-24, cleaved caspase-3, Beclin-1 and P62 antibody purchased from ProteinTech Group (UK). The protein levels of cleaved caspase-3, Beclin-1 and P62 were detected by enhanced chemiluminescence (ECL). $\beta$-actin was used as a loading control .

\section{Apoptosis analyses with flow cytometry}

Apoptosis was analyzed by flow cytometry, using annexin$\mathrm{V}$ and PI double staining following the manufacturer's protocol(Invitrogen, USA). Briefly, OSCC cells and control cells were cultured either with or without $3 \mathrm{mmol} / \mathrm{L} 3-\mathrm{MA}$ and with either AdLTR $_{2} E F 1 \alpha$-IL-24, AdLTR 2 EF1 $\alpha$-vec or virus dilution buffer. Two days later, cells were collected and washed with cold PBS and annexin-binding buffer. Then, annexin V and PI working solution were added cell suspension, then incubated at room temperature for $15 \mathrm{~min}$. After the incubation period, the stained cells were analyzed with flow cytometry.

\section{Measurement of caspase activity}

Caspase activity was measured using Caspase-Glo 3/7 assay kits (Promega, USA). Cells in each group were plated in opaque 96-well plates in triplicate. After $48 \mathrm{~h}$, the assay reagents were added to the plates at room temperature and incubated for $60 \mathrm{~min}$, and the fluorescence from the plates was then measured on a plate reader at $490 \mathrm{~nm}$ (excitation) and 510-570 nm (emission). Values were normalized to percentage of untreated control groups.

\section{In vitro cytotoxicity studies}

$\mathrm{KB}$ and $\mathrm{HaCaT}$ cells were treated with various $\mathrm{AdLTR}_{2} \mathrm{EF} 1 \alpha-$ based viruses (with or without 3-MA). Cells were incubated with $50 \mu \mathrm{l}$ of MTT solution $(5 \mathrm{mg} / \mathrm{ml})$ for $4 \mathrm{~h}$ at $37{ }^{\circ} \mathrm{C}$ at the indicated time points after treatment. After incubation, medium was removed in each well and replaced with $100 \mu \mathrm{l}$ Dimethyl sulfoxide (DMSO), then mixed thoroughly. Absorbance from the plates was read on a microplate reader at $490 \mathrm{~nm}$ wavelengths. The percentage of cell viability was calculated by multiplying the ratio absorbance of the sample versus the control by 100 .

\section{Cell cycle alteration}

$\mathrm{KB}$ and $\mathrm{HaCaT}$ cells were cultured in 6-well plates after transfection. After $48 \mathrm{~h}$, cells were harvested by trypsinization, washed in cold PBS, fixed with $70 \%$ ethanol at $-4{ }^{\circ} \mathrm{C}$ for $4 \mathrm{~h}$, and then were stained with propidium iodide (PI). DNA contents and cell cycle phases were analyzed using flow cytometry.

\section{Anticancer effect in vivo}

To determine whether the IL-24 gene and 3-MA would suppress the growth of tumor, twenty male Balb/c-nude mice (4-6 weeks old) were injected with KB cells $\left(1.5 \times 10^{6}\right.$ cells per mouse) in the dorsal flank to create subcutaneous tumors. When tumor size reached a predetermined size (50-80 $\mathrm{mm}^{3}$, about 8 days after the injection), mice were randomly divided into control, 3-MA, IL-24 and combination groups ( $n=5$ for each group). AdLTR ${ }_{2} E F 1 \alpha-I L-24$ (1010pfu/50ul) was injected around the tumor and 25per kilogram of 3-MA dissolved in 100ul saline was injected intraperitoneally every 6 days, a total of 3 times (day 6,12 , 18). As control, the same amount of saline was injected. Tumors were measured every 2 days and tumor volumes were calculated using the formula $V=1 / 2 \mathrm{ab}^{2}$ (where $a$ is the largest diameter and $b$ is the smallest diameter). Animals were observed for 30 days. After 1 month, tumor xenografts, hearts, livers and lungs were excised, routine fixed, paraffin-embedded, sliced and stained with HE. Terminal deoxynucleotidyl transferase-mediated biotinylate dUTPnick end labeling (TUNEL) assay was performed in the edge region of the tumor by using TUNEL Apoptosis Assay Kit-FITC according to the manufacturer's protocol (7 Sea Biotech). All manipulations involving living mice were approved by the Animal Care and Use Committee of the Harbin Institute of Technology.

\section{Statistical analysis}

Results are expressed as mean \pm SD. Statistical analysis was performed using the one-way analysis of variance and multiple comparison method, with $P<0.05$ deemed as statistically significant. All experiments were repeated at least three times.

\section{Results}

\section{Cytotoxicity and expression of the transgene}

As shown in Fig. 1a, at concentrations 250 to 1500pfu/ cell, cell viability of $\mathrm{KB}$ and $\mathrm{HaCaT}$ cells had no difference compared with the vector-free control group. In $2000 \mathrm{pfu} / \mathrm{cell}$ group and $2500 \mathrm{pfu} / \mathrm{cell}$ group, there was a slight decrease in cell viability compared with the vectorfree control group. Therefore, we selected $1000 \mathrm{pfu} / \mathrm{cell}$ as the appropriate concentration of $\mathrm{AdLTR}_{2} \mathrm{EF} 1 \alpha$-based vector with which to infect $\mathrm{KB}$ cells and $\mathrm{HaCaT}$ cells in subsequent experiments. As shown in Fig. 1b, more than $90 \%$ of EGFP expression was found in KB cells when infected with AdLTR $_{2} E F 1 \alpha$-EGFP. While in $\mathrm{HaCaT}$ cells EGFP expression level was about $15 \%$. The transgenic expression was determined by real time RT-PCR. As shown in Fig. 1c and d, a significant amount of IL-24 transcriptional expression was found in the AdLTR $_{2} E F 1 \alpha-I L-24$ treated $\mathrm{KB}$ and $\mathrm{HaCaT}$ cells, but IL-24 expression in the AdLTR $_{2} E F 1 \alpha$-vec treated and untreated groups was low. Furthermore, cells were infected, and then analyzed with Elisa Assay. As shown in Fig. 1e, We found the medium background was $361.33 \mathrm{ng} / \mathrm{L}$ in RPMI 1640 medium and 


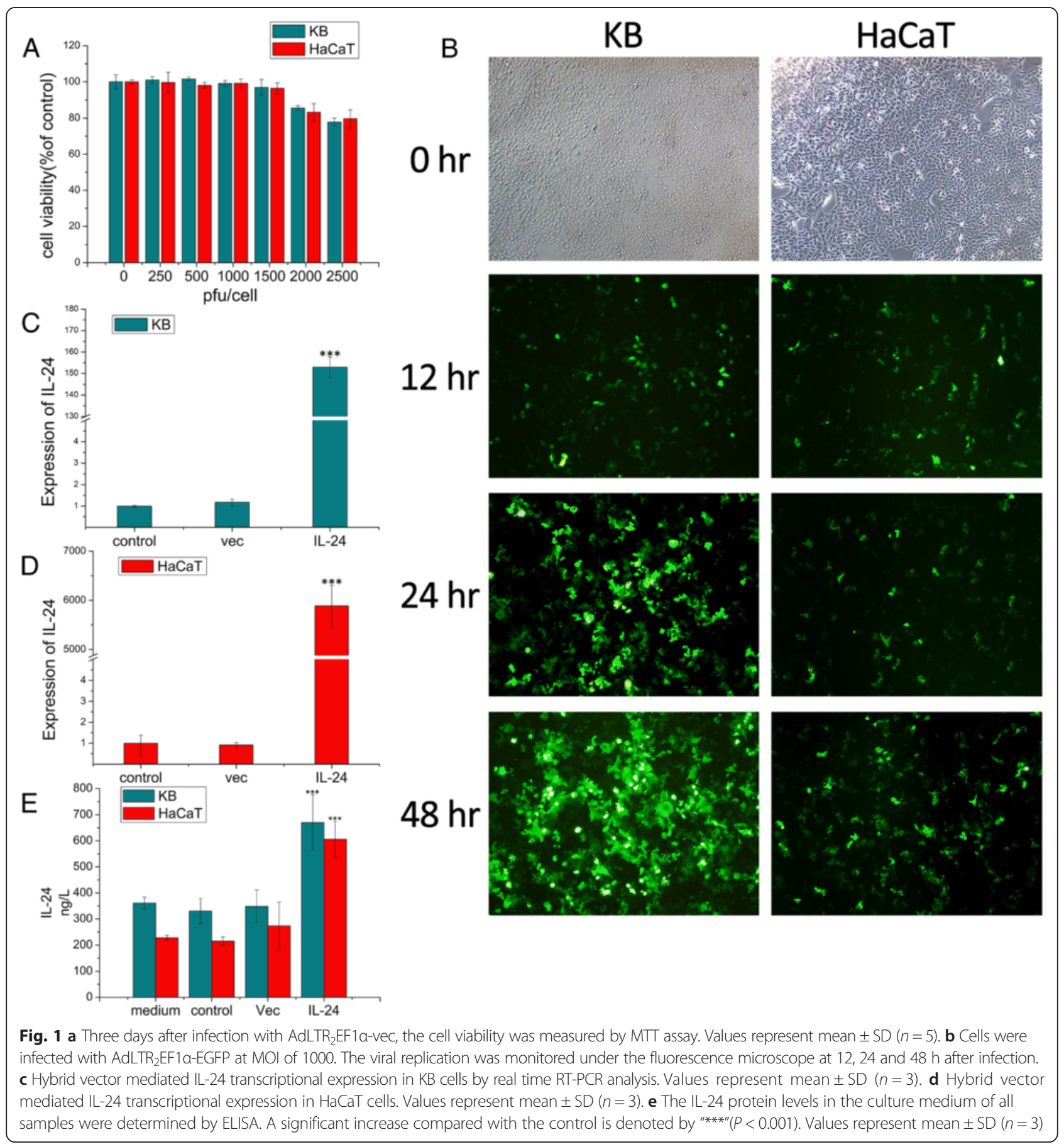

$227.48 \mathrm{ng} / \mathrm{L}$ in DMEM medium (all medium with $10 \%$ FCS). After subtracting the medium background, the date show that, IL-24 secretion in AdLTR ${ }_{2} E F 1 \alpha$-IL-24 treated cells was significantly higher than that of the control and AdLTR $_{2} \mathrm{EF} 1 \alpha$-vec treated groups.

These results suggest that transgenic IL-24 mediated by the vector was expressed in $\mathrm{KB}$ and $\mathrm{HaCaT}$ cells at both the transcriptional and translational levels.
AdLTR $_{2}$ EF1a-IL-24 induces autophagy in human OSCC cell line

The auto fluorescent drug MDC is a selective marker for autophagy vacuoles [13]. MDC accumulates in mature autophagy vacuoles, such as autophagosomes and autolysosomes. Autophagy was confirmed by MDCstaining of KB cells. As shown in Fig. 2a, AdLTR ${ }_{2} E F 1 \alpha-$ IL-24 treated cells showed an increasement in the number 


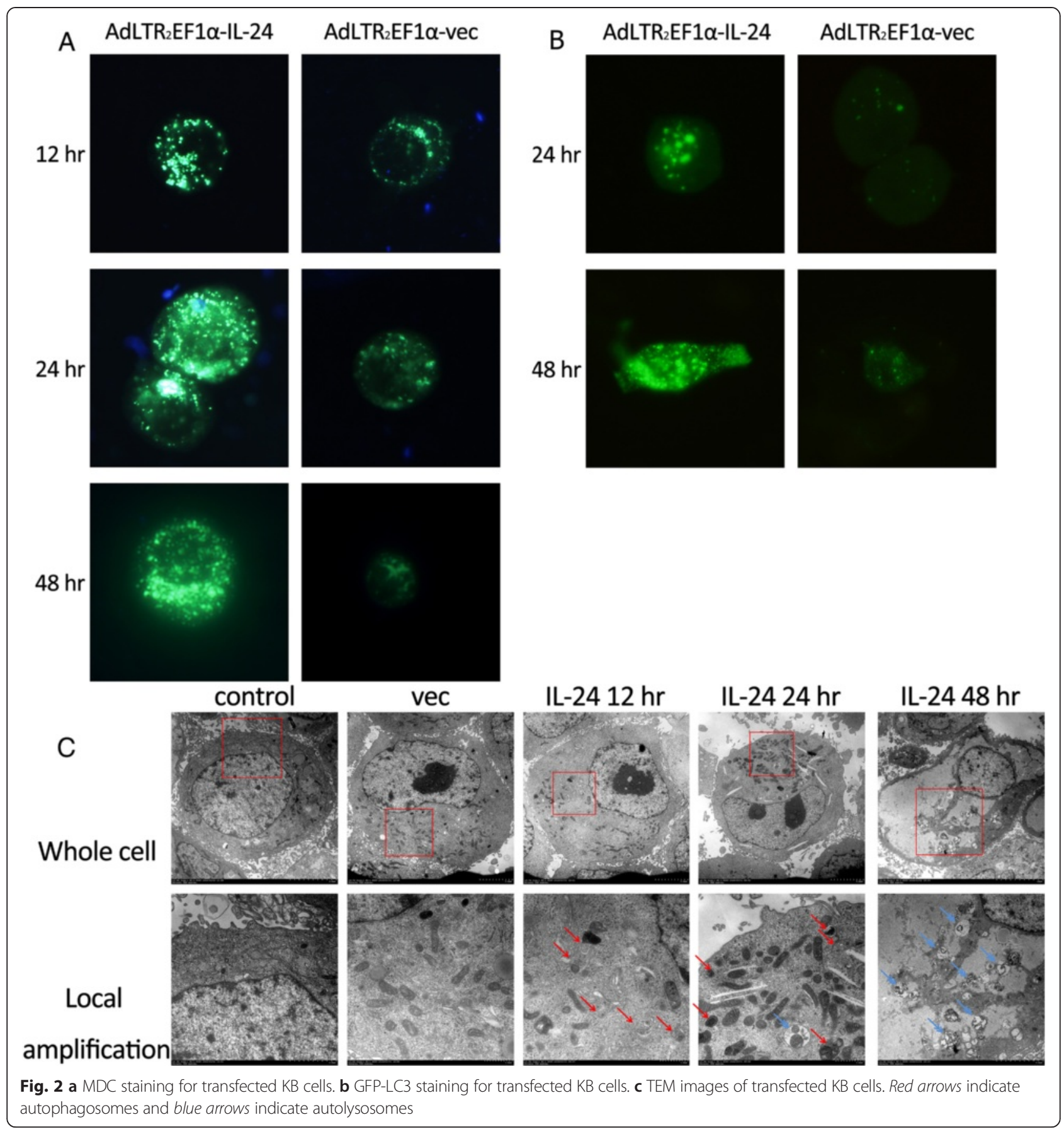

of MDC-labeled vacuoles compared to untreated cells, indicating that IL-24 induced the formation of the autophagosomes and autolysosomes. The localization of LC3 in autophagy vacuoles in the IL-24 treated KB cells was determined by transient transfection of a plasmid expressing green fluorescent protein fused with LC3 (GFP-LC3) followed by infection. Similar to MDC staining, after infection, GFP-LC3 staining increased in AdLTR $_{2}$ EF1 $\alpha$-IL-24 treated KB cells, when compared with the $\mathrm{AdLTR}_{2} \mathrm{EF} 1 \alpha-$ vec treated groups (Fig. 2b). TEM was used to detect autophagy vesicles with double membrane structure, also called autophagosomes. The existence of these vesicles is morphological evidence of autophagy. AdLTR ${ }_{2}$ EF1 $\alpha$-IL-24 induced a large number of autophagosomes in $\mathrm{KB}$ cells while the $\mathrm{AdLTR}_{2} \mathrm{EF} 1 \alpha$-vec treated or untreated KB cells showed low numbers of autophagosomes (Fig. 2c). These results indicate that $\mathrm{AdLTR}_{2} \mathrm{EF} 1 \alpha-\mathrm{IL}-24$ can strongly induce autophagy in $\mathrm{KB}$ cells. 
A

LC-3 II

$\begin{array}{llll}1 & 1.19 & 2.21 & 2.15\end{array}$

$\beta$-Tubulin

$0^{0}$

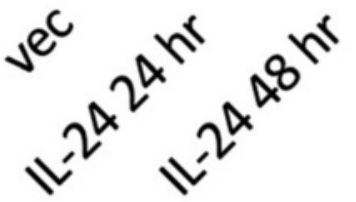

B

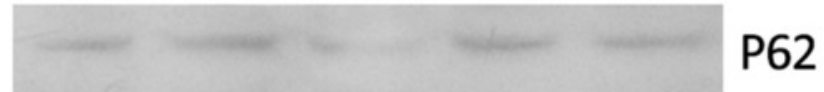

$=-2 m \ldots$ Beclin-1

Cleaved caspase-3
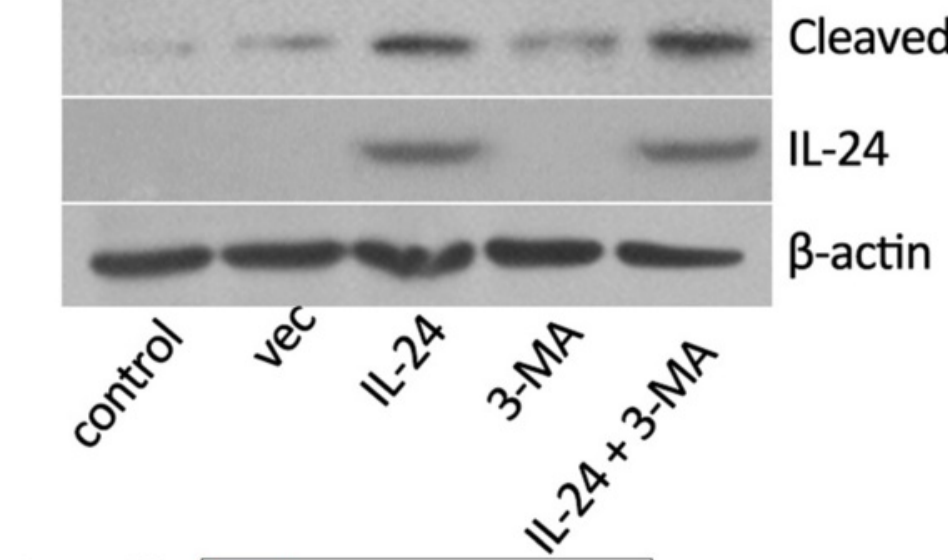

C

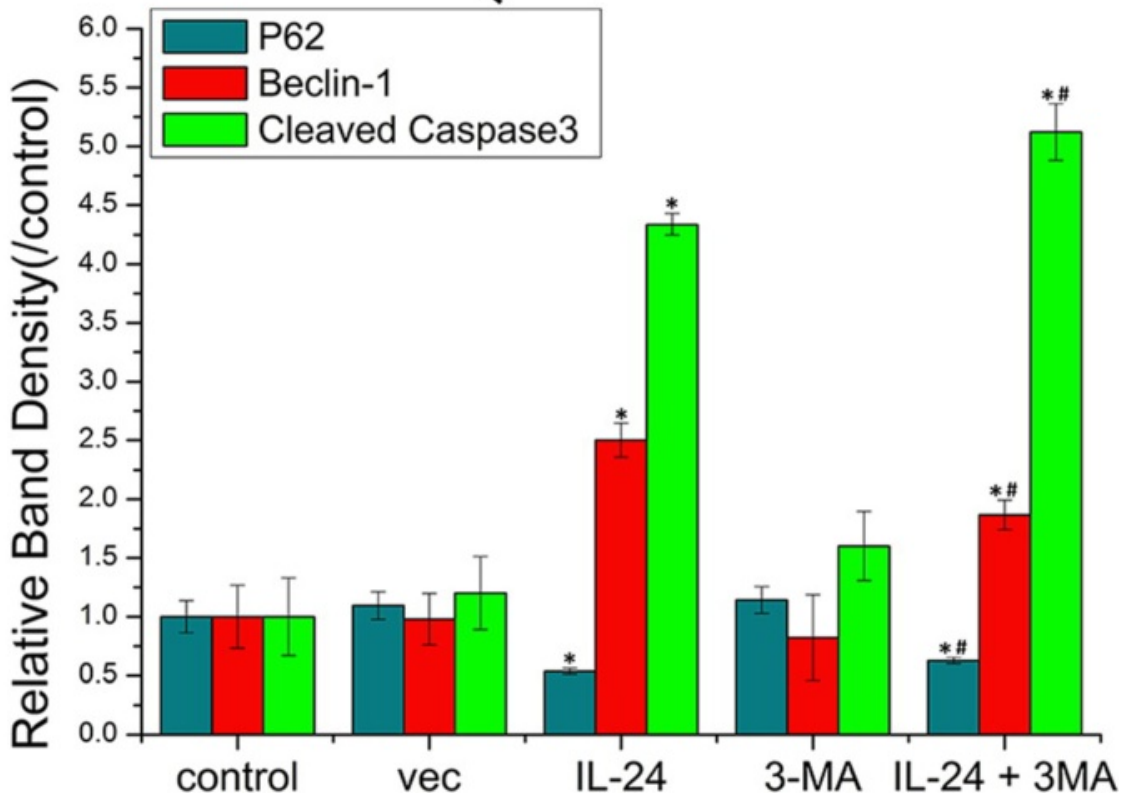

Fig. 3 (See legend on next page.) 
(See figure on previous page.)

Fig. 3 a KB cells were transfected and the level of LC3 was analyzed by Western immunoblotting. Densitometry was performed on the original blots, and the ratio of LC3-II/ $\beta$-tubulin in control cells was 1. b Western immunoblotting analysis of P62, Beclin-1, Cleaved caspase-3 and IL-24 in KB cells. c Semiquantitive analysis of P62, Beclin-1 and Cleaved caspase-3 in Kb cells. Compared with the control group denoted by "*" ( $p<0.05$ ). Compared with the IL-24-treated group denoted by "\#" $(p<0.05)$. Values represent mean $\pm \mathrm{SD}(n=3)$

\section{Western immunoblotting}

To further investigate the autophagy inducing effect of AdLTR $_{2} \mathrm{EF} 1 \alpha$-IL-24, cells were treated with different groups and the autophagy related proteins LC3-II, Beclin-1 and P62 were analyzed. As Fig. 3a shows, Infection of KB cells with AdLTR $_{2}$ EF1 $\alpha$-IL-24 led to an accumulation of LC3-II in a time-dependent manner when compared to the other groups. Moreover, treated KB cells showed an increase of Beclin-1 and decrease of P62 (Fig. 3b and c). Consistence with the results of MDC staining, GFP-LC3 transfection and TEM observation, these data also suggest that AdLTR $_{2}$ EF1 $\alpha$-IL-24 strongly induce autophagy in KB cells. Besides, to detect whether AdLTR $_{2}$ EF1 $\alpha$-IL-24 can lead to apoptosis, cells were treated by different groups and as a marker of apoptosis, the apoptosis related protein cleaved caspase- 3 was measured by western blot. As shown in Fig. 3b and c, the level of cleaved caspase-3 in combination of AdLTR $_{2}$ EF1 $\alpha$-IL-24 and 3-MA treated group was significantly up regulated compared to the other ones. This demonstrates that, AdLTR 2 EF1 $\alpha$-IL-24 can induce apoptosis in $\mathrm{KB}$ cells and the autophagy inhibitor 3-MA can enhance the apoptosis-inducing effect of AdLTR $_{2}$ EF $1 \alpha-I L-24$. Moreover, we also examine the level of IL-24 in KB cells treated with different groups. As Fig. 3b shows that in cells treated with IL-24 and IL-24 + 3-MA shows a high level of IL-24 and other groups express a low level of IL-24.

\section{IL-24 induced OSCC apoptosis was enhanced by 3-MA treatment}

As shown in Fig. 4a and b, the treatment of $\mathrm{KB}$ cells by AdLTR $_{2}$ EF1 $\alpha$-IL-24 caused $37.72 \%$ of cells apoptosis in the late and the early stage, whereas combining AdLTR $_{2}$ EF1 $\alpha$-IL-24 with 3 -MA resulted in $49.72 \%$ of cells apoptosis in the two stage total. Moreover, in the control group, the 3-MA group, the induced apoptotic percentages in $\mathrm{KB}$ cells are 16.64 and $18.09 \%$, respectively. However, in the human keratinocyte $\mathrm{HaCaT}$ cells, there were no apparent difference in all groups. Additionally, caspase-3/7 activities were increased obviously in the 3 -MA combining with AdLTR $_{2} E F 1 \alpha-I L-24$ group in $\mathrm{KB}$ cells. However, there were no significant changes in $\mathrm{HaCaT}$ cells in all of the groups (Fig. 4c). These results indicate that 3-MA enhance the IL-24 induced apoptosis.

\section{Effects on cell proliferation}

As shown in Fig. 5a, the cell viability of the control group is assumed to be $100 \%$. In KB cells, there was no significant retarding effect neither in the 3-MA group nor in the $\mathrm{AdLTR}_{2} \mathrm{EF} 1 \alpha-\mathrm{IL}-24$ group. In $\mathrm{AdLTR}_{2} \mathrm{EF} 1 \alpha-$ IL-24 group, the cell viability of $\mathrm{KB}$ cells was decreased to $69.05 \%$. However, in AdLTR $_{2}$ EF1 $\alpha$-IL-24 combining with 3-MA group, cell viability was reduced to $43.37 \%$. These data indicate that AdLTR 2 EF1 $\alpha$-IL-24 combining with 3-MA treatment inhibit the cell proliferation more effectively. But in $\mathrm{HaCaT}$ cells (Fig. 5b), no significant inhibition was observed in the IL-24, 3-MA or IL-24 combining with 3-MA groups (96.45\%, 97.74 and $94.80 \%)$. In addition, the cell cycles of $\mathrm{KB}$ cells (Fig. 5c) show higher levels of G2-M phase arrest in the IL-24 group (70.19\%), compared with the control group (25.04\%). The levels of G2-M phase arrest of the 3-MA group was similar to the control group (24.9\%). The IL-24 combining with 3-MA group showed the highest levels of G2-M arrest (88.85 \%). In HaCat cells (Fig. 5d), no significant difference in G2-M phase arrest was observed among the control, 3-MA and IL-24 groups (12.29, 12.85 and $15.50 \%)$, and there was slightly higher levels in the combination group (26.77\%), compared with the control group (24.9\%). These results demonstrated that AdLTR $_{2}$ EF1 $\alpha$-IL-24 selectively inhibits cancer cell proliferation by inducing G2-M cell cycle arrest, while there was no similar effect on normal cells. 3-MA treatment had no obvious effect on cell proliferation of normal and tumor cells. But AdLTR ${ }_{2}$ EF1 $\alpha$-IL-24 combining with 3-MA influenced the cell cycle of tumor cells strongly. However, the cell cycle of normal cells was just affected slightly by it.

\section{Antitumor efficacy of IL-24 combining with 3-MA}

To evaluate treatment effect, tumor-bearing mice were divided into four groups: control, 3-MA, IL-24 and IL-24 combining with 3-MA treatment group. All of the nude mice were healthy and body weight showed no significant difference in all groups (Fig. 6g).

Tumor volume and body weight of tumor-bearing mice were monitored every 2 days for 22 days. Compared with the control group, the tumor growth rate and size of IL24-treated group were significantly reduced and IL-24 combining with 3-MA group were further decreased (Fig. 6e and f). The 3-MA-treated group had no significant influence on tumor growth rate and size. After 30 days treatment, the mean tumor weight and mean tumor volumes of the IL-24 combining with 3-MA group were significantly reduced by 36.21 and $52.03 \%$, compared with the IL-24 group. HE staining of the tumors showed that, in the control group and 3-MA group, tumor cells grew more actively, their nuclei stained more deeply, they exhibited 


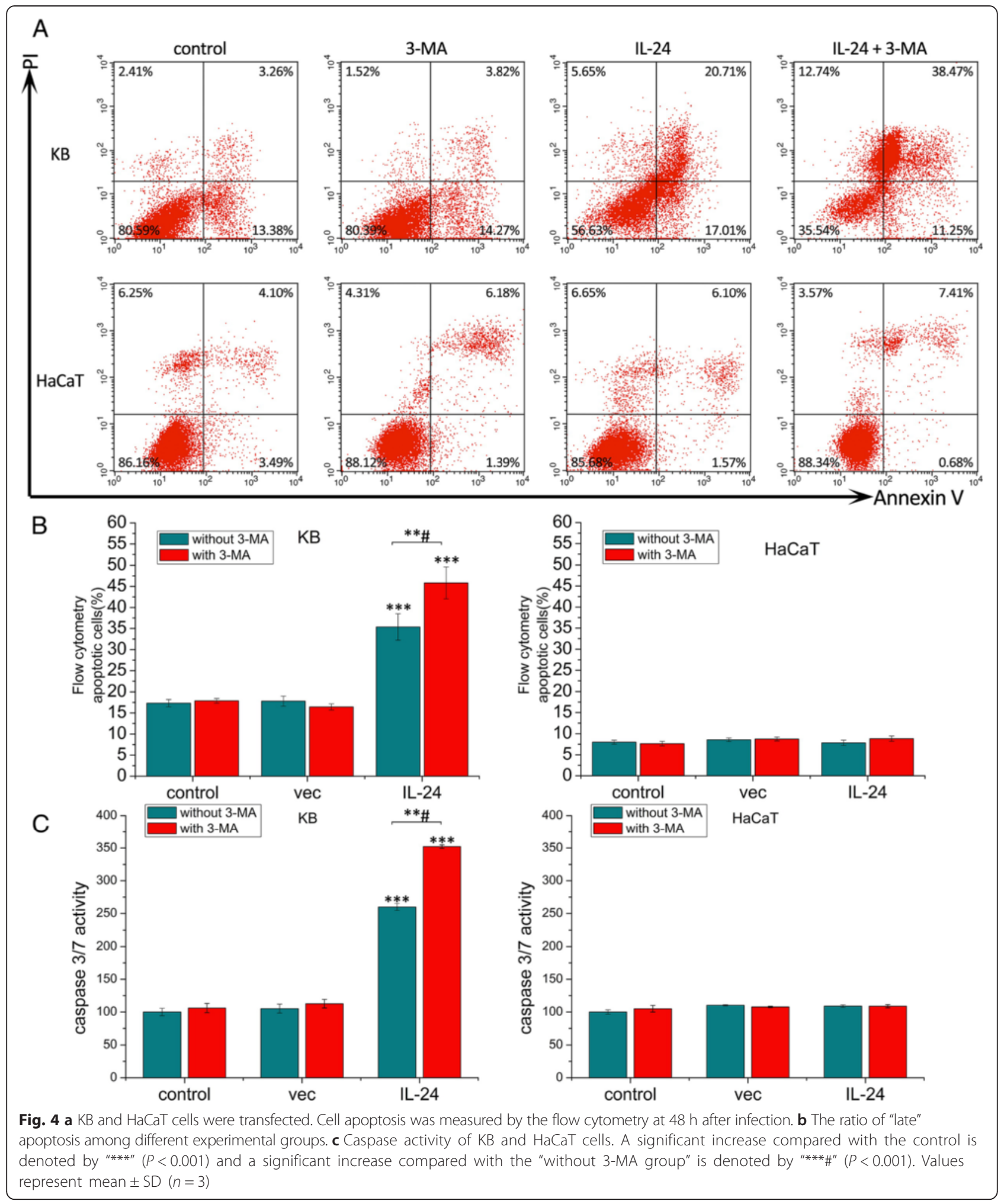

mitotic increase and necrotic cells are rare in these two groups. However, in the IL-24 group and the combination group tumor cells' growth was inhibited. However, there were a large number of necrotic cells, which have undergone karorrhexis (fragmentation) and karyolysis (dissolution) forming a uniform pink area, in the IL-24 group and the IL-24 combining with 3-MA group (Fig. 7a). Immunohistochemistry results showed that,we can found 


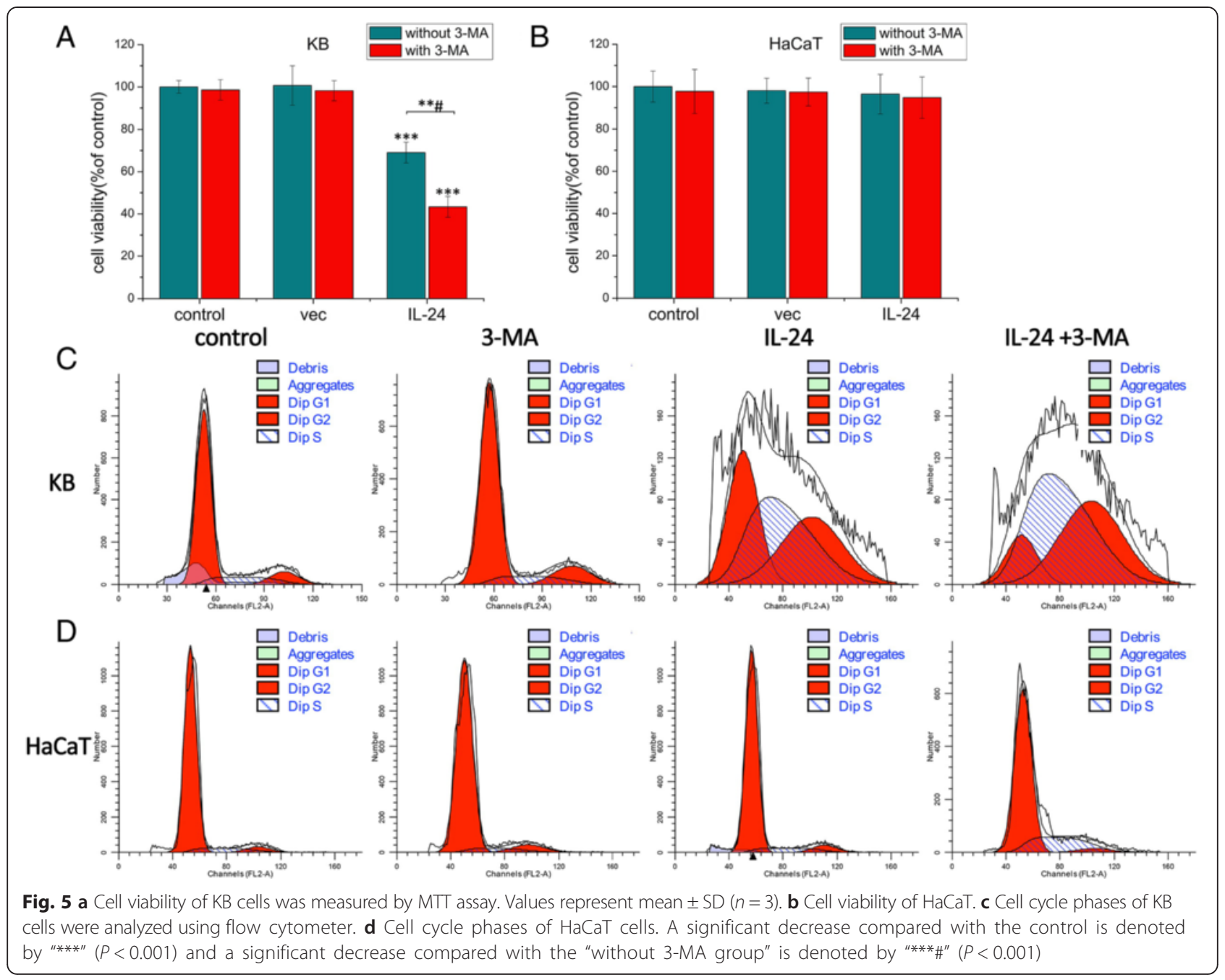

a high level of IL-24 protein in IL-24 group and IL-24 combining with 3-MA treated group. Whereas in the control group and 3-MA treated group, we found a low level of IL-24 protein (Fig. 7e and f). Hence, we confirm that tumor cells' growth was inhibited in the IL-24 group and the IL-24 combining with 3-MA group. Besides, there were no difference in the tissues of heart, liver and lung by HE staining (Fig. 7b, c and d). Moreover, IL-24 and IL24 combining with 3-MA group appeared increased number of TUNEL-positive cells (FITC-12-dUTP-Lable, green fluorescence). Whereas DNA fragmentation (measured by TUNEL assay) was rare in the control group and 3-MA group (Fig. $7 \mathrm{~g}$ and $\mathrm{h}$ ). These results prove that IL-24 has a strong antitumor effect in vivo and 3-MA improve the antitumor effect of it.

\section{Discussion}

Carcinomas of the oral cavity, particularly OSCC, have become an important health care problem worldwide. The survival rates for oral carcinomas are lower than those for most other carcinomas and have not improved substantially in recent years. The conventional treatment includes a combination of surgery, radiation therapy, and chemotherapy. Hence, to find a novel systemic therapy for OSCC is of great importance. Gene therapy of cancer has been taken into account as a potential therapeutic method.

Cancer gene therapy can be defined as the use of genetic material to manipulate tumor or normal cells to encourage anti-tumor activity such as direct killing of cells, immunomodulation or correction of genetic errors, and reversion of malignant status [14]. A successful cancer gene therapy requires an appropriate gene which displays selective toxicity toward tumor cells without eliciting harmful effects in normal cells or tissues. As a novel candidate for cancer gene therapy, IL-24 was cloned using subtraction hybridization from terminally differentiated human melanoma cells. Its unique properties include its ability to selectively induce growth suppression and apoptosis in diverse human cancer cells, without harmful effects on normal cells. Furthermore, the "potent 

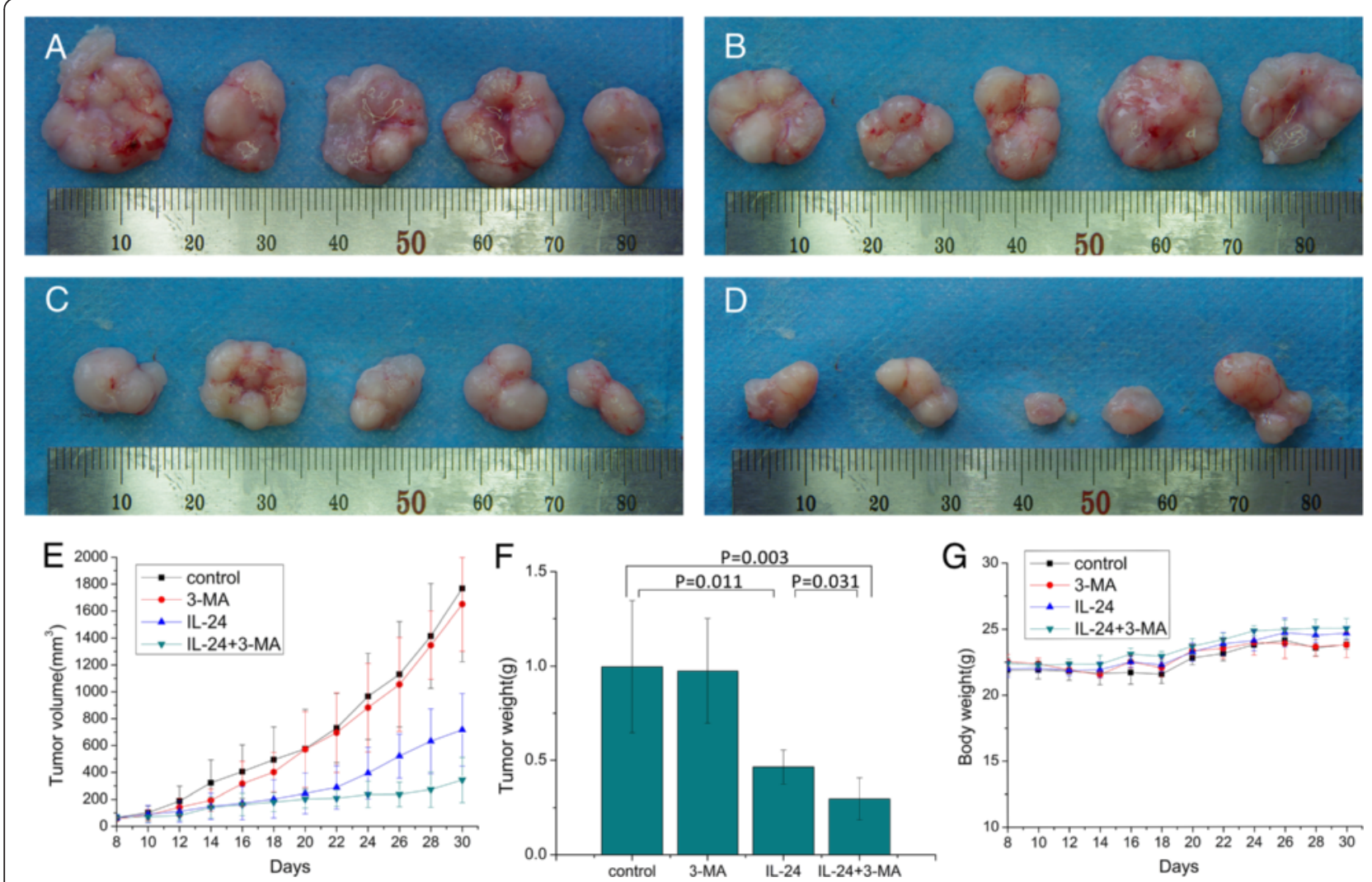

Fig. 6 a Removed KB tumor xenografts from the control group. b Xenografts from the 3-MA group. c Xenografts from the IL-24 group. d Xenografts from the combination group. e Tumor volume of KB xenografts. $\mathbf{f}$ Tumor weights of KB xenografts. $\mathbf{g}$ Body weights of the mice. Values represent mean \pm SD $(n=5)$

bystander apoptosis-inducing effect" is one of the antitumor properties of IL-24 [15]. IL-24 secreted by adjacent tumor or normal cells can induce direct antitumor effect in tumor cells not initially receiving this gene product.

However, a successful gene therapy also requires an appropriate kind of vectors. The ideal vectors should ensure a high level of transfection and should also maintain the gene expression for a suitable period of time and have no cytotoxicity. The two common viral vectors, based on adenovirus or retrovirus, have advantages and drawbacks. Traditional adenovirus has advantages including ease of production, high titers, efficient transduction into many types of cells, and infrequent genomic integration [16, 17]; But also has the shortcomings of provoking vigorous immune responses and only providing shortterm transgenic expression (14 days) [18]. The period of time transgenic expression provided by retrovirus vectors is longer than that of adenovirus vector, but their transduction requires cell division, and genomic integration may be uncontrolled, thereby introducing the risk of insertional mutagenesis $[19,20]$.

The viral vector used in this study is an adenovirusretrovirus hybrid vector, named $\mathrm{AdLTR}_{2} \mathrm{EF} 1 \alpha$-base vector. It can be transfected into a variety of cells, and can provide intermediate-length (1-3 months) expression of the gene, with low frequency of genomic integration. Tumor cells or normal cells infected with AdLTR $_{2}$ EF1 $\alpha$-IL-24 inhibit the surrounding tumor cells, that not initially infected with AdLTR $_{2} E F 1 \alpha-I L-24$, through the "bystander" effect. The intermediate-length expression ofAdLTR ${ }_{2} E F 1 \alpha$-IL-24 is conducive to exerting the anti-tumor "bystander" effect. Thus the AdLTR $_{2} E F 1 \alpha$-base vector is an ideal vector for cancer gene therapy.

Our studies demonstrated that AdLTR $R_{2}$ EF1 $\alpha$-IL-24 induces not only $\mathrm{KB}$ cells but also $\mathrm{HaCaT}$ cells to express IL-24 highly. In KB cells, infection with $\mathrm{AdLTR}_{2} \mathrm{EF} 1 \alpha-$ IL-24 induced a high level of apoptosis, whereas the AdLTR $_{2} E F 1 \alpha$-vec did not have such effects. However, in $\mathrm{HaCaT}$ cells, high level of IL-24 did not lead to obvious increasement of apoptosis. It indicates that IL-24 only harmful to tumor cells and almost have no damage to normal cells. Thus AdLTR $_{2}$ EF1 $\alpha$-IL-24 may become a new therapeutic agent for cancer gene therapy, given the intermediate-length expression and targeted induction of apoptosis.

Autophagy is a catabolic process. Cells employ autophagy to recycle basic biomolecules during nutrient deprivation, to scavenge damaged organelles and harmful proteins, and 


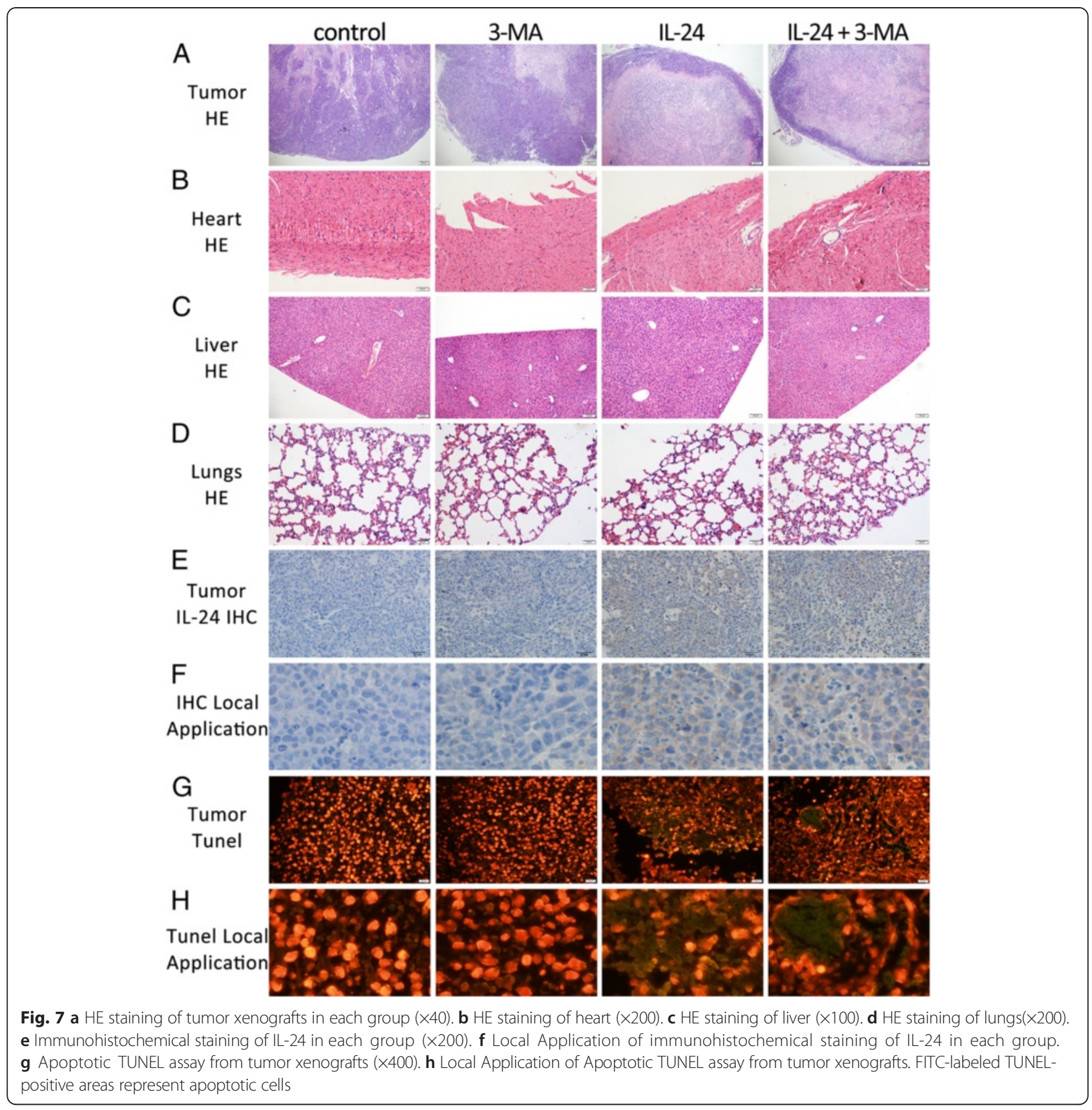

to eliminate intracellular pathogens. The process of autophagy plays a crucial role in maintaining cellular homeostasis, and also contributes to cell survival during times of stress [21]. Although apoptosis and autophagy constitute different cellular processes and generally cause opposite results, their signaling pathways are interconnected by a wide variety of mechanism crosstalk. It is a key factor in deathrelated pathologies such as cancer [22]. In cell, such a crosstalk is synergistic, accomplished by a variety of mechanisms including the ATG5-ATG12 conjugation [23], the Beclin 1-VPS34 complex [24], as well as caspase-8 and autophagic cargo receptor p62 interaction [25]. In this study, we confirmed that AdLTR $_{2} E F 1 \alpha$-IL-24 induced autophagy of KB cells by MDC staining, GFP-LC3 staining, western immunoblotting and transmission electron microscope observation. We consider that autophagy is a self-protection mechanism against IL-24 induced apoptosis in cancer cells. Inhibition of autophagy in tumor cells has been reported to enhance the effect of chemotherapy and to increase the cytotoxicity induced by drug. One of the most important effects of cytotoxicity is apoptosis [26]. To identify whether autophagy inhibition can improve the apoptosis-inducing effect of IL-24, a well known autophagy inhibitor 3MA was introduced. The results from MTT assays, flow 
cytometric and caspase-3/7 activity assays demonstrated that the autophagy inhibitor 3-MA can strongly enhance the anticancer effect of IL-24.

However, although 3-MA can enhance the antitumor effect of IL-24 in vitro, whether it is also effective in vivo is need to be investigated. In this study we established $K B$ xenografts models and treat the mice with 3-MA, IL-24 and the combination of 3-MA and IL-24. Our data indicate that tumor volume and mass of the IL-24 treated group was significantly less than that of the 3-MA treated group and control group, while the combination of 3-MA and IL-24 group was even less than the IL-24 group. HE staining and TUNEL experiments further confirmed these results. Consistent with the results of in vitro experiments, these data indicate that AdLTR $_{2} E F 1 \alpha-I L-24$ not only has antitumor effects in vitro, but also has similar effects in vivo, and autophagy inhibition can enhance the antitumor effect of AdLTR ${ }_{2} \mathrm{EF} 1 \alpha$-IL-24 both in vitro and in vivo.

\section{Conclusion}

This study has demonstrated that IL-24 carried by AdLTR $_{2} E F 1 \alpha$ vector has a strong anticancer effect towards OSCC in both in vitro and in vivo experiments. Autophagy, as a self-protective mechanism, was induced by IL-24 treatment and the autophagy inhibition by 3-MA significantly enhance the anticancer effect of IL-24. Therefore, we believe that the strategy of combination of IL-24 and autophagy inhibitor will open up new perspectives in clinically applicable combination therapy to treat OSCC.

\begin{abstract}
Abbreviations
OSCC: Oral squamous cell carcinoma; GFP-LC3: Green fluorescent protein fused with LC3; 3-MA: 3-methyladenine; LL-24: Interleukin-24; mda-7: Melanoma differentiation-associated gene-7; KB: Human Oral epidermoid cancer cells; HaCaT: Immortal human keratinocyte cells; EGFP: Enhanced green fluorescent protein; real time RT-PCR: Real time reverse-transcription polymerase chain reaction; ELISA: Enzyme-linked immunosorbent assay; MDC: Monodansylcadaverine; TEM: Transmission electron microscope; ECL: Enhanced chemiluminescence; DMSO: Dimethyl sulfoxide; PI: Propidium iodide; TUNEL: Terminal deoxynucleotidyl transferase-mediated biotinylate dUTPnick end labeling.
\end{abstract}

\section{Competing interests}

The authors declare that they have no competing interests.

\section{Authors' contributions}

$Y L, H S$ and JL: conceptualized the project. JL, DY, SP, WS, CZ, HS, and YL: participated in the study design. $J \mathrm{~L}$ and DY: Performed the biological experiments. JL, DY, WS: composed the manuscript. JL, WW, JZ: quality control and statistical analysis. YL and HS: interpreted the data, gave important intellectual input toward the introduction, results and discussion. All co-authors: read and edited the manuscript drafts as well as gave final approval of the final manuscript draft.

\section{Acknowledgement}

We thank Ronald C. Inglehart for helpful discussions and assistance with writing. This work was supported by the Returned Student Science Fund (LC2009C04) of Heilongjiang province and National Natural Science Foundation Committee of International Cooperation Bureau (30672338), People's Republic of China.

\section{Author details}

'School of Life Science and Technology, Harbin Institute of Technology, 2 Yikuang Street, Harbin 150001, People's Republic of China. ${ }^{2}$ Department of Oral and Maxillofacial Surgery, School of Dentistry, Harbin Medical University, 141 Yiman Street, Nangang District, Harbin 150001, People's Republic of China. ${ }^{3}$ National Institute of Dental and Craniofacial Research, National Institutes of Health, Bethesda, MD, USA. ${ }^{4}$ Department of Oral Pathology, School of Stomatology, Jilin University, 1500 Qinghua Road, Changchun 130021, People's Republic of China.

Received: 11 February 2015 Accepted: 25 August 2015

Published online: 11 September 2015

\section{References}

1. Wikner J, Gröbe A, Pantel K, Riethdorf S. Squamous cell carcinoma of the oral cavity and circulating tumour cells. World J Clin Oncol. 2014;5(2):114.

2. Chada S, Sutton RB, Ekmekcioglu S, Ellerhorst J, Mumm JB, Leitner WW, et al. MDA-7/IL-24 is a unique cytokine-tumor suppressor in the IL-10 family. Int Immunopharmacol. 2004;4(5):649-67.

3. Zhu W, Wei L, Zhang H, Chen J, Qin $X$, et al. Oncolytic adenovirus armed with IL-24 inhibits the growth of breast cancer in vitro and in vivo. J Exp Clin Cancer Res. 2012;31(1):51.

4. Lebedeva IV, Emdad L, Su ZZ, Gupta P, Sauane M, Sarkar D, et al. mda-7/IL-24, novel anticancer cytokine: focus on bystander antitumor, radiosensitization and antiangiogenic properties and overview of the phase I clinical experience (Review). Int J Oncol. 2007;31(5):985-1007.

5. Altman BJ, Rathmell JC. Autophagy: not good or bad, but good and bad. Autophagy. 2009;5:569-70.

6. Kumar P, Zhang D-M, Degenhardt K, Chen Z-S. Autophagy and transporterbased multi-drug resistance. Cell. 2012;1:558-75.

7. Zhang Q, Si S, Schoen S, Chen J, Jin X-B, Guan W. Suppression of autophagy enhances preferential toxicity of paclitaxel to folliculin-deficient renal cancer cells. J Exp Clin Cancer Res. 2013;32(1):99.

8. Xi G, Hu X, Wu B, Jiang H, Young CY, Pang Y, et al. Autophagy inhibition promotes paclitaxel-induced apoptosis in cancer cells. Cancer Lett. 2011;307:141-8.

9. Tu YJ, Fan X, Yang X, Zhang C, Liang HP. Evodiamine activates autophagy as a cytoprotective response in murine Lewis lung carcinoma cells. Oncol Rep. 2013;29:481-90.

10. Sheen JH, Zoncu R, Kim D, Sabatini DM. Defective regulation of autophagy upon leucine deprivation reveals a targetable liability of human melanoma cells in vitro and in vivo. Cancer Cell. 2011;19:613-28.

11. Park MA, Yacoub A, Sarkar D, Emdad L, Rahmani M, Spiegel S, et al. PERK-dependent regulation of MDA-7/IL-24-induced autophagy in primary human gliomacells. Autophagy. 2008;4(4):513.

12. Zheng C, Vitolo JM, Zhang W, Mineshiba F, Chiorini JA, Baum BJ. Extended transgene expression from a nonintegrating adenoviral vector containing retroviral elements. Mol Ther. 2008;16(6):1089-97.

13. Biederbick A, Kern HF, Elsässer HP. Monodansylcadaverine (MDC) is a specific in vivo marker for autophagicvacuoles. Eur J Cell Biol. 1995;66(1):3-14.

14. Mitrović T, Radulović S. Cancer gene therapy. Arch Oncol. 2005;13(1):23-6.

15. Su Z, Emdad L, Sauane M, Lebedeva IV, Sarkar D, Gupta P, et al. Unique aspects of mda-7/IL-24 antitumor bystander activity: establishing a role for secretion of MDA-7/IL-24 protein by normal cells. Oncogene. 2005;24(51):7552-66.

16. Greber UF, Webster P, Weber J, Helenius A. The role of the adenovirus protease on virus entry into cells. EMBO J. 1996;15(8):1766.

17. Harui A, Suzuki S, Kochanek S, Mitani K. Frequency and stability of chromosomal integration of adenovirus vectors. J Virol. 1999;73(7):6141-6.

18. Jooss K, Chirmule N. Immunity to adenovirus and adeno-associated viral vectors: implications for gene therapy. Gene Ther. 2003;10(11):955-63.

19. Miller DG, Adam MA, Miller AD. Gene transfer by retrovirus vectors occurs only in cells that are actively replicating at the time of infection. Mol Cell Biol. 1990;10(8):4239-42.

20. Baum C, Kustikova O, Modlich U, Li Z, Fehse B. Mutagenesis and oncogenesis by chromosomal insertion of gene transfer vectors. Hum Gene Ther. 2006;17(3):253-63.

21. Levine B, Kroemer G. Autophagy in the pathogenesis of disease. Cell. 2008;132(1):27-42. 
22. Eisenberg-Lerner A, Bialik S, Simon HU, Kimchi A. Life and death partners: apoptosis, autophagy and the cross-talk between them. Cell Death Differentiation. 2009;16(7):966-75.

23. Rubinstein $A D$, Eisenstein $M$, Ber $Y$, Bialik S, Kimchi $A$. The autophagy protein Atg12 associates with antiapoptotic Bcl-2 family members to promote mitochondrial apoptosis. Mol Cell. 2011:44(5):698-709.

24. Maiuri MC, Le Toumelin G, Criollo A, Rain JC, Gautier F, Juin P, et al. Functional and physical interaction between $\mathrm{BCl}-\mathrm{XL}$ and a $\mathrm{BH}$-like domain in Beclin-1. EMBO J. 2007;26(10):2527-39.

25. Young MM, Takahashi Y, Khan O, Park S, Hori T, Yun J, et al. Autophagosomal membrane serves as platform for intracellular death-inducing signaling complex (iDISC)-mediated caspase-8 activation and apoptosis. J Biol Chem. 2012:287(15):12455-68.

26. Kumar S, Yedjou CG, Tchounwou PB. Arsenic trioxide induces oxidative stress, DNA damage, and mitochondrial pathway of apoptosis in human leukemia (HL-60) cells. J Exp Clin Cancer Res. 2014;33(1):42.

\section{Submit your next manuscript to BioMed Central and take full advantage of:}

- Convenient online submission

- Thorough peer review

- No space constraints or color figure charges

- Immediate publication on acceptance

- Inclusion in PubMed, CAS, Scopus and Google Scholar

- Research which is freely available for redistribution 